\title{
Enriching Constraints and Business Rules in Object Oriented Analysis Models with Trigger Specifications
}

\author{
Stefan Van Baelen \\ K.U.Leuven, Department of Computer Science \\ Celestijnenlaan 200A, B-3001 Leuven, BELGIUM \\ Stef an. VanBaelenecs.kuleuven.ac.be \\ WWW home page: http://ww.cs.kuleuven.ac.be/som/
}

\section{Motivation}

Current object oriented analysis methods focus especially on class centered information and inter-object behavior, expressed in static structural and object interaction diagrams. The specification of constraints and business rules ${ }^{1}$ is not a major concern to them. Although UML (Unified Modeling Language) provides support for con-straint specifications through OCL (Object Constraint Language), its integration with other model concepts is rather minimal. Constraints are treated as formal comment specifications rather than distinct and important model elements. For instance, the interaction between constraints and object behavior is often neglected. It is not clear how a message that violates a certain constraint can be refused without crashing the whole system. UML states that the condition of a constraint must always be true, otherwise the system is invalid with consequences outside the scope of UML. As such, constraints are not really imposed on a model and its behavior, but serves only as a validation of a model state at a certain moment in time.

To highlight the importance of constraints in analysis models, they should be treated as first-class model elements with a clear semantic impact on existing static and dynamic model elements. As such, validation and verification of constraints becomes possible. Due to their broadness in nature, constraints can have a local or a global impact on a model. A single constraint can delimit certain functionality of a large number of involved classes, or can have a focussed impact on a specific attribute or association.

Several kinds of reaction patterns for constraint violation should be supported. It is not suitable for a system to end up in an inconsistent model state after a constraint violation. Optimal constraint support includes concepts for specifying event refusal, event transformation and event triggering mechanisms

\footnotetext{
${ }^{1}$ With Constraint/Business Rule, we mean rules defined on the problem domain, restricting certain events or services or forcing certain business policies. It describes normal or wanted situations in the problem domain, excluding exceptional, not allowed or alarm situations. Derivation rules are outside the scope of this position paper.
} 
in order to deal with possible constraint violations. Realization of constraints during design can be obtained through the introduction of constraint checker objects or the transformation of declarative constraint specifications into operational behavior restrictions and checks.

\section{Specifying Constraints as first-class Model Elements}

The semantic impact of constraints on a model is so that each instantiation of the static structural model over time must comply with the specified constraints. This means that a system will never arrive in an unsafe state, unless the specified model is faulty and inconsistent. As a consequence, the dynamic model is restricted so that behavioral elements - such as events, messages and methods - cannot occur unless they preserve all constraints. A method that causes a constraint violation must raise an exception or is refused instead of being executed. As such, the analyst can rely on the constraints and correctness of the system at any moment in time. Notice that this rely on the detection of possible constraint violations in order to be able to refuse method execution, or on the presence of a rollback mechanism to undo the previous effects of a method violating certain constraints.

\section{Specifying Triggers as a Reaction Mechanism for Constraint Violations}

In past experiments, we noticed that a method refusal mechanism was not adequate for most cases to avoid constraint violation. In fact, an object that is planning to call a method often knows that this method can cause a constraint violation, and therefore tries to anticipate this by investigating the system state and avoiding a constraint violation. On the one hand, this introduces a lot of unwanted overhead for a method call setup and duplication of constraint checking code. On the other hand, such approach is impossible to manage and maintain on a longer term.

To overcome these problems, we extended the constraint definition with optional trigger specifications, defining the reaction of the system on a constraint violation. Each constraint can be extended with a number of event specifications that will only fire when the constraint is actually violated. These events can be defined on a general level, on a specific caller object, on a method causing a violation or on the actual parameters of a method.. As such, the specification of the anticipation of a constraint violation is not a part of the caller or the callee, but is an integral part of the constraint itself. The semantic meaning of a trigger in reaction to a constraint violation can be made in two distinct ways.

- On the one hand, an addition trigger can be performed after the method that violates the constraint. As such, the trigger must try to resolve the constraint violation by reforming the system state into a valid one. This can for instance be done by extending certain deadlines or creating certain credit notes. 
- On the other hand, a replacement trigger refuses the whole method causing the constraint violation. Instead it will transform the method call into another call that is conform to the applicable constraints. This can for instance be applied when a certain service is replaced with a newer one, changing the old service call into a call on the new service. Another example is when a unauthorized person request a certain service. The constraint that enforces the authorization can transform the service call into a registration of the authorization violation into the database. Although this is a different kind of reaction pattern, both reactions can be specified using a method replacement trigger for a constraint violation. 